\title{
Erratum to: A prospective study on predicting local recurrence of giant cell tumour of bone by evaluating preoperative imaging features of the tumour around the knee joint
}

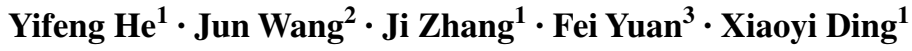

Published online: 1 April 2017

(C) Italian Society of Medical Radiology 2017

\section{Erratum to: Radiol med}

DOI 10.1007/s11547-017-0745-7

In the original publication, the first and last names of the fourth author were interchanged. The correct name of the author should read as "Fei Yuan".

The original article has been updated accordingly.

The online version of the original article can be found under doi:10.1007/s11547-017-0745-7.

Xiaoyi Ding

dingxiaoyi@hotmail.com

Yifeng $\mathrm{He}$

hyff86@163.com

Jun Wang

wwwjjj815@163.com

Ji Zhang

614757632@qq.com

Fei Yuan

daphny2014@163.com

1 Department of Radiology, Ruijin Hospital, Shanghai Jiao Tong University School of Medicine, 197, Rui Jin Er Road, Shanghai 200025, China

2 Shanghai Institute of Traumatology and Orthopaedics, Shanghai Key Laboratory for Prevention and Treatment of Bone and Joint Diseases, Ruijin Hospital, Shanghai Jiao Tong University School of Medicine, Shanghai, China

3 Department of Pathology, Ruijin Hospital, Shanghai Jiao Tong University School of Medicine, Shanghai, China 